Probability, Networks and Algorithms

P Probability, Networks and Algorithms

J. van den Berg, R.M. Brouwer

Report PNA-R0303 MARCh 31, 2003 
CWI is the National Research Institute for Mathematics and Computer Science. It is sponsored by the Netherlands Organization for Scientific Research (NWO).

CWI is a founding member of ERCIM, the European Research Consortium for Informatics and Mathematics.

CWI's research has a theme-oriented structure and is grouped into four clusters. Listed below are the names of the clusters and in parentheses their acronyms.

\section{Probability, Networks and Algorithms (PNA)}

Software Engineering (SEN)

Modelling, Analysis and Simulation (MAS)

Information Systems (INS)

Copyright (C) 2001, Stichting Centrum voor Wiskunde en Informatica

P.O. Box 94079, 1090 GB Amsterdam (NL)

Kruislaan 413, 1098 SJ Amsterdam (NL)

Telephone +31205929333

Telefax +31205924199

ISSN 1386-3711 


\title{
Self-destructive Percolation
}

\author{
J. van den Berg and R.M. Brouwer \\ $C W I$
}

P.O. Box 94079, 1090 GB Amsterdam, The Netherlands

\begin{abstract}
Consider ordinary site percolation on an infinite graph in which the sites, independent of each other, are occupied with probability $p$ and vacant with probability $1-p$. Now suppose that, by some 'catastrophe', all sites which are in an infinite occupied cluster become vacant. Finally, each vacant site gets an extra enhancement to become occupied. More precisely, each site that was already vacant or that was made vacant by the catastrophe, becomes occupied with probability $\delta$ (independent of the other sites). When $p$ is larger than but close to the critical value $p_{c}$ one might believe (for 'nice' graphs) that only a small $\delta$ is needed to have an infinite occupied cluster in the final configuration. This appears to be indeed the case for the binary tree. However, on the square lattice we strongly conjecture that this is not true. We discuss the background for these problems and also show that the conjecture, if true, has some remarkable consequences.
\end{abstract}

2000 Mathematics Subject Classification: 60K35

Keywords and Phrases: percolation, continuity properties, forest fire models

Note: This report has been submitted for publication elsewere. This work has been done under project PNA 3.1 .

\section{INTRODUCTION}

The square lattice is the graph whose sites (vertices) are the points of $\mathbb{Z}^{2}$, and where two sites $i$ and $j$ are neighbours (share an edge) if $|i-j|=1$. We perform independent site percolation on this graph, i.e. we declare each site occupied with probability $p$, and vacant with probability $1-p$, independent of the other sites.

For general information and results on percolation theory, see Grimmett (1999).

Let, as usual, $\theta(p)$ denote the probability that a given site, say $O=(0,0)$, belongs to an infinite occupied cluster. It is known that there is a critical value $0<p_{c}<1$ such that $\theta(p)>0$ if and only if $p>p_{c}$.

Now suppose that, by a catastrophe, each site which is in an infinite occupied cluster is destroyed (that is, becomes vacant). (For this lattice, and more generally on $\mathbb{Z}^{d}, d \geq 2$, there is, if $p>p_{c}$, a.s. a unique infinite occupied cluster, but on general graphs there can be more than one). Further suppose that, after this catastrophe, we give the sites independent 'enhancements'. More precisely, each site that after the above catastrophe was vacant (i.e. not only the sites that were originally, before the catastrophe, in an infinite occupied cluster but also those that were originally vacant) becomes occupied with probability $\delta$, independent of the others (and those that were occupied after the catastrophe reamain occupied).

Let $\mathcal{P}_{p, \delta}$ denote the distribution of the final configuration. Usually we write 1 for occupied and 0 for empty so that we consider $\mathcal{P}_{p, \delta}$ as a distribution on $\{0,1\} \mathbb{Z}^{d}$ (with the natural $\sigma$-field). Further, we use the notation $\theta(p, \delta)$ for the probability that, in the final configuration, $O$ is in an infinite occupied cluster:

$$
\theta(p, \delta):=\mathcal{P}_{p, \delta}(O \rightarrow \infty)
$$


To get some feeling for the above definitions: it is easy to check that $O$ is occupied in the final configuration if and only if the above mentioned enhancement was successful, or $O$ belonged initially (before the catastrophe) to a non-empty but finite occupied cluster. This gives

$$
\mathcal{P}_{p, \delta}(O \text { is occupied })=\delta+(1-\delta)(p-\theta(p) .
$$

Also, in the case that $p \leq p_{c}$, nothing happens in the above catastrophe ( since then $\theta(p)=0$ ), so that in the final configuration the sites are independently occupied with probability $p+(1-p) \delta$. Formally, if $p \leq p_{c}$, then

$$
\mathcal{P}_{p, \delta}=\mathcal{P}_{p+(1-p) \delta},
$$

where we use the notation $\mathcal{P}_{p}$ for the product measure with parameter $p$.

In particular,

$$
\theta\left(p_{c}, \delta\right)=\theta\left(p_{c}+\left(1-p_{c}\right) \delta\right)>0,
$$

for each $\delta>0$.

It also immediately clear from the construction that if $\delta>p_{c}$, then $\theta(p, \delta)>0$ for all $p$.

It takes a little more effort to prove that, if $p>p_{c}$, a 'non-negligible' enhancement is needed after the catastrophe to reintroduce an infinite occupied cluster. More precisely, for each $p>p_{c}$ there is a $\delta>0$ with $\theta(p, \delta)=0$. This is proved in Proposition 3.1. A much more difficult question is whether the needed effort to reintroduce an infinite occupied cluster after the catastrophe, goes to 0 as $p \downarrow p_{c}$. By (1.1) one might be tempted to intuitively reason that this is indeed the case. However, based on arguments described in Section 3, we strongly believe that this is false: we conjecture that there is a $\delta>0$ for which $\theta(p, \delta)=0$ for all $p>p_{c}$. (See Theorem 3.3 and Conjecture 3.2). For those who think that this is not surprising at all: for the similar model on the binary tree we can prove that the above mentioned intuitive reasoning gives a correct conclusion: for that model we prove in Section 5 that

$$
\lim _{p \downarrow p_{c}} \theta(p, \delta)=\theta\left(p_{c}, \delta\right)>0 .
$$

Although we mainly restrict to the square lattice and the binary tree, it is convenient for our purpose to describe the model in some more generality. This, together with the presentation of some basic properties, will be done in Section 2.

\section{GENERALISATION OF THE MODEL, AND BASIC RESULTS}

\subsection{Generalisation}

We will now put the above model in a more general context. Let $G$ be a finite or countably infinite graph, $V(G)$ the set of vertices of $G$ and $\Gamma$ is either a subset of $V(G)$ or the symbol $\infty$. Let $p, \delta \in[0,1]$. Analogously to what we did in Section 1 , we first make each vertex $i$, independent of the other vertices, occupied with probability $p$ and vacant with probability $1-p$. Then, in the next step, we destroy all occupied clusters intersecting $\Gamma$ (when $\Gamma=\infty$ we destroy the infinite occupied clusters). That is, each vertex which has an occupied path to $\Gamma$, is made vacant. Finally, in the last step, each vertex which is vacant at the end of the previous step, is made occupied with probability $\delta$. The probability distribution of the final configuration will be denoted by $\mathcal{P}_{p, \delta}^{(G ; \Gamma)}$. Often, when the choice of $G$ is obvious, it is omitted from the notation. Also, when $\Gamma=\infty$ we will usually omit it from the notation.

A more formal, and often more convenient for our purposes, description of the model is as follows: Let $X_{i}, i \in V(G)$ be independent $0-1$ valued random variables, each $X_{i}$ being 1 with probability $p$ and 0 with probability $1-p$. Further, Let $Y_{i}, i \in V(G)$ be independent $0-1$ valued random variables, each $Y_{i}$ being 1 with probability $\delta$ and 0 with probability $1-\delta$. Moreover, we take the collection of $Y_{i}$ 's independent of that of the $X_{i}^{\prime}$ 's. Let $X_{i}^{*}=X_{i}^{*}(G, \Gamma)$ be defined by 


$$
X_{i}^{*}=\left\{\begin{array}{l}
1 \text { if } X_{i}=1 \text { and there is no } X \text { - occupied path from } i \text { to } \Gamma \\
0 \text { otherwise, }
\end{array}\right.
$$

where by ' $X$-occupied path' we mean a path on which each site $j$ has $X_{j}=1$.

Finally, define $Z_{i}=X_{i}^{*} \vee Y_{i}$. Then $\mathcal{P}_{p, \delta}^{(G ; \Gamma)}$ is the distribution of the collection $\left(Z_{i}, i \in V(G)\right)$.

\subsection{Association}

Recall that a finite collection of random variables $\left(\omega_{i}, 1 \leq i \leq n\right.$ ) (or its corresponding probability measure, say $\mu$, on $\left.\{0,1\}^{n}\right)$ is said to be associated if for all increasing events $A, B \subset\{0,1\}^{n}$,

$$
\mu(A \cap B) \geq \mu(A) \mu(B) .
$$

This is equivalent to saying that for all increasing functions $f$ and $g$ on $\{0,1\}^{n}$,

$$
E_{\mu}(f g) \geq E_{\mu}(f) E_{\mu}(g),
$$

where

$$
E_{\mu}(f)=\sum_{x \in\{0,1\}^{n}} \mu(x) f(x)
$$

Lemma 2.1. Let $G$ be a finite graph, and let $\Gamma, p$ and $\delta$ be as above. Then $\mathcal{P}_{p, \delta}^{(G ; \Gamma)}$ is associated.

Proof. The following facts (a)-(c) are well-known.

(a) A collection of independent random variables is associated.

(b) If a collection $\left(\omega_{i}, 1 \leq i \leq n\right)$ is associated, and a collection $\left(\sigma_{i}, 1 \leq i \leq m\right)$ is associated, and these two collections are independent of each other, then the joint collection $\left(\omega_{i}, 1 \leq i \leq n ; \sigma_{j}, 1 \leq j \leq m\right)$ is associated.

(c) If a collection $\left(\omega_{i}, 1 \leq i \leq n\right)$ is associated, and $f_{1}, \cdots, f_{k}$ are increasing $0-1$-valued functions on $\{0,1\}^{n}$, then the collection $\left(f_{1}\left(\omega_{1}, \cdots \omega_{n}\right), \cdots, f_{k}\left(\omega_{1}, \cdots, \omega_{n}\right)\right)$ is associated.

Now let the random variables $X_{i}, Y_{i}, X_{i}^{*}$ and $Z_{i}$ be as defined in the previous subsection. From the definition of these random variables, and the above mentioned facts it is immediately clear that, to prove the lemma, it is sufficient to prove that the collection $X_{i}^{*}, i \in V(G)$ is associated. This is proved as follows First some more notation. First let $\Omega=\{0,1\}^{V(G)}$. For $\omega \in \Omega$ we let $C=C(\Gamma, \omega)$ be the occupied cluster of $\Gamma$ (i.e. the set of all sites which have a path to $\Gamma$ on which $\omega \equiv 1$ ).

For $W \subset V(G), \bar{W}$ denotes the set which consists of $W$ and all neighbours of $W$.

If $W \subset V(G)$ and $A \subset \Omega$ is increasing, the event ( $A$ occurs outside $W$ ) will denote the set of all $\omega \in \Omega$ such that the configuration $\omega^{\prime}$, defined by

$$
\omega_{i}^{\prime}=\left\{\begin{array}{l}
0 \text { if } i \in W \\
\omega_{i} \text { otherwise }
\end{array}\right.
$$

is in $A$.

Let $\mu_{p}$ be the product measure with parameter $p$ on $\Omega$, and let $\mu_{p}^{*}$ be the measure on $\Omega$ corresponding with the the collection $\left(X_{i}^{*}, i \in V(G)\right)$.

We have 


$$
\begin{aligned}
\mu_{p}^{*}(A \cap B) & =\sum_{W} \mu_{p}(\overline{C(\Gamma)}=W, A \cap B \text { occurs outside } W) \\
& =\sum_{W} \mu_{p}(\overline{C(\Gamma)}=W) \mu_{p}(A \cap B \text { occurs outside } W) \\
& \geq \sum_{W} \mu_{p}(\overline{C(\Gamma)}=W) \mu_{p}(A \text { occurs outside } W) \mu_{p}(B \text { occurs outside } W)
\end{aligned}
$$

where we have summed over all subsets $W$ of $V(G)$, and where we used that the event $\{\overline{C(\Gamma)}=W\}$ depends only on the sites in $W$, and that the events $\{A$ occurs outside $W\}$ and $\{B$ occurs outside $W\}$ are increasing (so that we can use the association property of $\mu_{p}$ ). Now let, for $W \subset V(G)$, $f(W)$ denote $\mu_{p}(A$ occurs outside $W)$ and let $g(W)$ denote $\mu_{p}(B$ occurs outside $W)$. It is clear that if $W \subset W^{\prime}$, then $f(W) \geq f\left(W^{\prime}\right)$, and similar for $g$. So the last expression above equals

$$
\begin{aligned}
& \sum_{W} \mu_{p}(\overline{C(\Gamma)}=W) f(W) g(W) \\
= & \sum_{W} \sum_{\omega \in \Omega: \overline{C(\Gamma, \omega)}=W} \mu_{p}(\omega) f(W) g(W) \\
= & \sum_{\omega \in \Omega} \mu_{p}(\omega) f(\overline{C(\Gamma, \omega)}) g(\overline{C(\Gamma, \omega)}) \\
\geq & \sum_{\omega \in \Omega} \mu_{p}(\omega) f(\overline{C(\Gamma, \omega)}) \sum_{\omega \in \Omega} \mu_{p}(\omega) g(\overline{C(\Gamma, \omega)}) \\
= & \mu_{p}^{*}(A) \mu_{p}^{*}(B),
\end{aligned}
$$

where the inequality holds because $f(\overline{C(\Gamma, \omega)})$ and $g(\overline{C(\Gamma, \omega)})$ are both decreasing in $\omega$ (so that we can apply the association property of $\mu_{p}$ ), and the last equality follows from similar arguments as before (but now 'working backwards').

\subsection{Weak convergence}

Let $G$ be a countably infinite, locally finite graph, $W$ a subset of the vertices of $G$, and $A \subset\{0,1\}^{W}$. Fix a site $O$ of $G$, and let $D(n)$ denote the set of all vertices at (graph) distance $\leq n$ from $O$. Let $\partial D(n)$ be the external boundary of $D(n)$, i.e. $\partial D(n)=D(n+1) \backslash D(n)$. Recall the definition (in the beginning of this section) of the distribution $\mathcal{P}_{p, \delta}^{(G ; \partial D(n))}$.

\section{Lemma 2.2 .}

$$
\lim _{n \rightarrow \infty} \mathcal{P}_{p, \delta}^{(G ; \partial D(n))}(A)=\mathcal{P}_{p, \delta}^{G}(A)
$$

Proof. Using the description of the $X$ and $Y$ variables in Subsection 2.1, it is easy to see that, if $n$ is sufficiently large (so that $W \subset D(n)), \mathcal{P}_{p, \delta}^{(G ; \partial D(n))}(A)$ and $\mathcal{P}_{p, \delta}^{G}(A)$ differ at most the probability that there is an $i \in W$ that has an $X$-occupied path to $\partial D(n)$ but does not have an infinite $X$-occupied path. This is clearly at most

$$
\sum_{i \in W} \mathcal{P}_{p}(i \rightarrow \partial D(n))-\mathcal{P}_{p}(i \rightarrow \infty) .
$$

Since for each $i$ the term in the above summation goes to 0 , and $W$ is finite, the lemma follows.

Remark: By combining the two lemma's it follows that $\mathcal{P}_{p, \delta}^{G}$ is associated. 


\subsection{Monotonicity}

It is immediately clear from the definitions that $\mathcal{P}_{p, \delta}$ is stochastically increasing in $\delta$. Strictly speaking there is no monotonicity in $p$. However, we will show that 'some sort of such monotonicity' does hold: Let $0 \leq p<q \leq 1$. Let $\Gamma$ be a subset of the vertices (or $\infty$ ).

Lemma 2.3. For fixed $q$, the family of probability measures $\mathcal{P}_{p,(q-p) /(1-p)}^{(G ; \Gamma)}$ is stochastically decreasing in $p<q$.

Remark: By the observation made a few lines before the lemma, it is clear that the family of probability measures in the lemma is (for fixed $p$ ) stochastically increasing in $q$.

Proof. Consider the following time evolution. At time 0 each site of $G$ is vacant. Sites become, independent of each other, occupied at rate 1. If this would simply go on, then, clearly, for each $t \geq 0$, the distribution of the configuration at time $t$ is exactly $\mathcal{P}_{1-\exp (-t)}$. Now suppose that at a deterministic time $T$ a 'catastrophe' happens: all sites that have, at that time, an occupied path to $\Gamma$, become (instantaneously) vacant. From then on the process behaves 'normally' again, i.e. vacant sites become occupied at rate 1 . It follows from the following coupling argument, that, for fixed $t>0$, the configuration at time $t$ is stochastically decreasing in the time $T(T<t)$ at which the catastrophe takes place:

Let $U_{i}^{(k)}, i \in V(G), k \in\{1,2\}$ be i.i.d. exponentially distributed random variables with mean $1 . U_{i}^{(1)}$ denotes the times at which $i$ becomes occupied for the first time. If a site is made vacant by the catastrophe at time $T$, then it becomes occupied again at time $T+U_{i}^{(2)}$. More formally, if we let $\eta_{i}(t)$ denote the state (occupied or vacant) of $i$ at time $t$, then we have for all $t>T: \eta_{i}(t)=0$ iff $U_{i}^{(1)}>t$ or $\left(U_{i}^{(2)}>t-T\right.$ and there is a path $\pi$ from $i$ to $\Gamma$ with $U_{j}^{(1)}<T$ for all sites $j$ on $\left.\pi\right)$. It is immediately clear that if this condition holds for some $T<t$, then it also holds for all $T^{\prime} \in(T, t)$.

It is also clear from the description above that the distribution of $\left(\eta_{i}(t), i \in V(G)\right)$ is $\mathcal{P}_{1-\exp (-T), 1-\exp (-(t-T))}^{(G)}$. This, together with the monotonicity observation we just made, gives that, for fixed $t$, the family of distributions

$$
\mathcal{P}_{1-\exp (-T), 1-\exp (-(t-T))}^{(G ; \Gamma)}, 0 \leq T<t
$$

is stochastically decreasing in $T$. The Lemma now follows by taking $t$ and $T$ such that $p=1-\exp (-T)$ and $q=1-\exp (-t)$ (and hence $(q-p) /(1-p)=1-\exp (-(t-T)))$.

An immediate consequence of this lemma (and the obvious monotonicity of $\mathcal{P}_{p, \delta}$ in $\delta$ ) is the following:

Corollary 2.4. If $p_{2} \geq p_{1}$ and $p_{2}+\left(1-p_{2}\right) \delta_{2} \leq p_{1}+\left(1-p_{1}\right) \delta_{1}$, then

$$
\mathcal{P}_{p_{1}, \delta_{1}} \text { dominates } \mathcal{P}_{p_{2}, \delta_{2}} \text {. }
$$

\section{CONTINUITY OR DISCONTINUITY OF $\theta(p, \delta)$}

In this section we focus on site percolation on the square lattice, although most of what we say also holds for many other two-dimensional lattices.

It is intuitively plausible that if $p>p_{c}$ and we destroy the infinite cluster, we need some minimal (depending on $p$ ) positive enhancement to reintroduce an infinite cluster. This is indeed the case, as stated more formally by the following proposition:

\section{Proposition 3.1.}

$$
\forall p>p_{c} \exists \delta>0, \text { s.t. } \theta(p, \delta)=0 .
$$




\section{Remarks:}

(i) Note that, by earlier mentioned (obvious) monotonicity, it follows that for all $\delta^{\prime}$ smaller than the $\delta$ in this proposition $\theta\left(p, \delta^{\prime}\right)=0$.

(ii) In fact we prove a much more explicit statement (see below). This proof can be done in practically the same way for site percolation on the triangular lattice. For bond percolation on the square lattice the analog of the proposition still holds, but the proof is somewhat more complicated (and we don't know an analog of the more explicit claim below).

Proof. We claim that

$$
\text { if } p(1-\delta)>p_{c} \text {, then } \theta(p, \delta)=0 \text {. }
$$

From this the proposition immediately follows. To prove this claim, we use the $X$ and $Y$ variables introduced in subsection 2.1. Colour each site $i$ red if $X_{i}=1$ and $Y_{i}=0$. So, each site is, independent of the others, red with probability $p(1-\delta)$. Now suppose the pair $p, \delta$ satisfies the condition in the claim. Then, by standard results from 'ordinary' site percolation on the square lattice, there is (a.s.) an infinite red cluster, and this cluster contains arbitrary large red contours (loops) around $O$. Each site $j$ on such a contour has $X_{j}^{*}=0$ (because it is in an infinite red, and hence $X$-occupied, cluster) and $Y_{j}=0$ and hence $Z_{j}=0$. Hence, a.s. there is no infinite path from $O$ on which $Z \equiv 1$. So $\theta(p, \delta)$ is indeed 0 .

As said before, the Proposition above is intuitively plausible. With a similar intuition one might also be tempted to guess the following: Consider the distribution $\mathcal{P}_{p, \delta}$. In particular consider the description in Section 1. Since (as is well-known) $\theta(p) \downarrow \theta\left(p_{c}\right)=0$ as $p \downarrow p_{c}$, we have that, if $p$ is larger than but very close to $p_{c}$, only a very small portion (namely $\theta(p)$ ) of the sites is effected by the 'catastrophe'. Therefore it is reasonable to believe that then only a very small 'enhancement' is needed to regain an infinite occupied cluster. More formally this belief would state that the answer to the following question is true (and thus would give in some sense a counterpart to the Proposition $3.1)$.

\section{Question:}

Does the following hold?

$$
\forall \delta \exists p>p_{c} \text { s.t. } \theta(p, \delta)>0 .
$$

Remark: One might even go further and wonder if

$$
\lim _{p \downarrow p_{c}} \theta(p, \delta)=\theta\left(p_{c}, \delta\right),
$$

which, as we observed before, equals $\theta\left(p_{c}+\left(1-p_{c}\right) \delta\right)$ and hence is positive (so that (3.3) would follow).

In fact, we believed for a while that this is indeed the case. (And we can prove that for the binary tree this statement is correct; see Section 5). But now we strongly conjecture that for regular planar lattices including the square lattice, (3.3) is not true.

The rest of this section is meant to relate this problem to quite naturally defined numbers (probabilities) $a_{n}(\delta)$. We will prove that if for some $\delta>0$ the sequence $\left(a_{n}(\delta)\right)$ is bounded away from 1 , then the answer to the Question above is negative.

We now will define the numbers $a_{n}$ mentioned above. Let $n>0$. Consider the box $[0,3 n] \times[0,2 n]$. By inserting the edges inherited from the square lattice, we consider this as a graph, denoted by $G=G(n)$. Let $L=L(n)$ be the lower side $\{(i, 0): 0 \leq i \leq 3 n\}$ of the box, $M=M(n)$ the horizontal middle line $\{(i, n): 0 \leq i \leq 3 n\}$, and $U=U(n)$ be the upper side $\{(i, 2 n): 0 \leq i \leq 3 n\}$ of the box. Then $a_{n}=a_{n}(\delta)$ is formally (using the notation in Section 2.1) defined as follows: 


$$
a_{n}:=\mathcal{P}_{p_{c}, \delta}^{(G ; U)}(L \rightarrow M)
$$

Based on computer simulations we strongly believe the following:

\section{Conjecture 3.2.}

$\exists \delta>0$ s.t. the sequence $\left(a_{n}(\delta)\right)$ is bounded away from 1.

Theorem 3.3. If for some $\delta>0$ the sequence of probabilities $\left(a_{n}(\delta)\right)$ is bounded away from 1 , then

$$
\exists \delta>0 \text { s.t. } \forall p>p_{c} \theta(p, \delta)=0 .
$$

To prove the theorem we need a few lemmas and introduce some notation. We define

$$
\begin{gathered}
B(n)=[-n, n] \times[-n, n], n \geq 0 . \\
S(n)=B(n) \backslash B(n-1), n>0,
\end{gathered}
$$

i.e. $S(n)$ is the boundary of $B(n)$. Further,

$$
A(n, m)=B(m) \backslash B(n-1) .
$$

In additions to the $a_{n}$ 's above we now define $b_{n}$ 's as follows: Consider the annulus $A(n, 5 n)$. First make each site occupied with probability $p$. Then make vacant each site whose occupied cluster in $A(3 n, 5 n)$ contains a contour around $B(3 n)$. Finally, make a $\delta$-enhancement as usual. We define $b_{n}=b_{n}(p, \delta)$ as the probability that the final configuration has an occupied path from $S(n)$ to $S(3 n)$. For simplicity we will often write 'contour in $A(3 n, 5 n)$ ' for 'contour around $B(3 n)$ in $A(3 n, 5 n)$ '.

Lemma 3.4. If $\delta$ is such that the sequence $\left(a_{n}(\delta)\right)$, defined by $(3.4)$, is bounded away from 1 , then also the sequence $\left(b_{n}\left(p_{c}, \delta\right)\right)$ is bounded away from 1 .

Proof. In this proof we will write $a_{n}$ for $a_{n}(\delta)$ and $b_{n}$ for $b_{n}\left(p_{c}, \delta\right)$. We claim that

$$
\begin{aligned}
b_{n} \leq \quad & \mathcal{P}_{p_{c}}(\text { there is no occupied contour in } A(3 n, 5 n)) \\
+ & \left(1-\left(1-a_{2 n}\right)^{4}\right) \mathcal{P}_{p_{c}}(\exists \text { occupied contour in } A(3 n, 5 n)) .
\end{aligned}
$$

The Lemma follows from this claim and the well-known fact (RSW theorem) that $\mathcal{P}_{p_{c}}(\exists$ occupied contour around $A(3 n)$ in $A(3 n, 5 n))$ is bounded away from 0 .

Proof of claim: Analogously to what we did earlier, let $X_{i}, i \in A(n, 5 n)$ be i.i.d. Bernoulli random variables with parameter $p$, and $Y_{i}, i \in A(n, 5 n)$ i.i.d. Bernoulli random variables with parameter $\delta$, and define, for $i \in A(n, 5 n)$, the following random variables in terms of the $X$ and $Y$ variables:

$$
\begin{aligned}
& X_{i}^{(* c)}:=\quad I\left(X_{i}=1 \text { but the } X \text {-occupied cluster of } i \text { in } A(n, 5 n)\right. \\
&\text { contains no contour around } B(3 n)) . \\
& X_{i}^{(* S)}:=I\left(X_{i}=1 \text { but } \nexists X \text { - occupied path in } A(n, 5 n) \text { from } i \text { to } S(5 n)\right),
\end{aligned}
$$




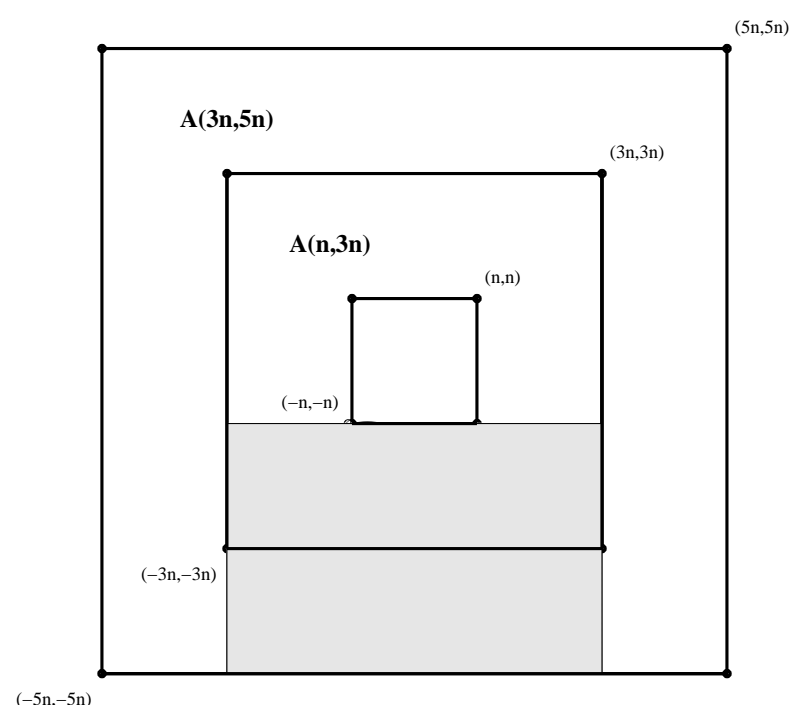

Figure 1: The annuli $A(n, 3 n)$ and $A(3 n, 5 n)$. The shaded region is the rectangle $R$.

where $I(\cdot)$ denotes the indicator function.

Further, let

$$
Z_{i}^{(c)}:=X_{i}^{(* c)} \vee Y_{i}
$$

and

$$
Z_{i}^{(S)}:=X_{i}^{(* S)} \vee Y_{i}
$$

It is clear that for each $i \in A(n, 3 n)$, each path from $i$ to $S(5 n)$ intersects each contour in $A(3 n, 5 n)$ around $B(3 n)$. From this and the above definitions it follows immediately that if there is an $X-$ occupied contour in $A(3 n, 5 n)$, then, for all $i \in A(n, 3 n), X_{i}^{(* c)} \leq X_{i}^{(* S)}$. Hence

$$
\begin{aligned}
& b_{n}=\mathcal{P}\left(\exists Z^{(S)}-\text { occupied path from } S(n) \text { to } S(3 n)\right) \\
\leq & \mathcal{P}(\nexists X-\text { occupied contour in } A(3 n, 5 n)) \\
+ & \mathcal{P}\left(\exists X-\text { occupied contour in } A(3 n, 5 n) ; \exists Z^{(S)}-\text { occupied path from } S(n) \text { to } S(3 n)\right) \\
\leq & \mathcal{P}(\nexists X-\text { occupied contour in } A(3 n, 5 n)) \\
+ & \mathcal{P}(\exists X-\text { occupied contour in } A(3 n, 5 n)) \\
& \times \mathcal{P}\left(\exists Z^{(S)}-\text { occupied path from } S(n) \text { to } S(3 n)\right),
\end{aligned}
$$

since the event $\{\exists X$ - occupied contour in $A(3 n, 5 n)\}$ depends only on the variables $X_{i}, i \in A(3 n, 5 n)$ (and is increasing in those variables), while the event $\left\{\exists Z^{(S)}\right.$ - occupied path from $S(n)$ to $\left.S(3 n)\right\}$ is obviously decreasing in those variables (so that we can use the FKG inequality).

Let $R_{1}, \cdots, R_{4}$ be the four $2 n \times 6 n$ rectangles whose union is $A(n, 3 n)$. To be more explicit, let $R_{1}$ be the lowest of the two horizontal rectangles. Denote the two long sides of $R_{1}$ by $L_{1}$ and $L_{2}$, where $L_{2}$ is the bottom. Let $R$ denote the union of $R_{1}$ and its mirror image under reflection in $L_{2}$. (see figure 1).

Finally, let $L_{3}$ be the bottom side of $R$ (so $L_{3}$ is the set $\{(i,-5 n):-3 n \leq i \leq 3 n\}$ ). Now, in our earlier notation, the factor

$$
\mathcal{P}\left(\exists Z^{(S)} \text { - occupied path from } S(n) \text { to } S(3 n)\right)
$$


in the r.h.s. of $(3.9)$ is

$$
\mathcal{P}_{p, \delta}^{(A(n, 5 n) ; S(5 n))}(S(n) \rightarrow S(3 n)) .
$$

By the association property (Lemma 2.1) and the (well-known and often used) fact that a path from $S(n)$ to $S(3 n)$ always contains a subpath in one of the four rectangles $R_{1}, \cdots, R_{4}$, between the two long sides of that rectangle, and by symmetry (each of the four rectangles 'plays the same role'), this is at most

$$
1-\left(1-\mathcal{P}_{p, \delta}^{(A(n, 5 n) ; S(5 n))}\left(L_{1} \rightarrow L_{2} \text { in } R_{1}\right)\right)^{4},
$$

which by an obvious monotonicity argument is at most

$$
1-\left(1-\mathcal{P}_{p, \delta}^{\left(R ; L_{3}\right)}\left(L_{1} \rightarrow L_{2} \text { in } R_{1}\right)\right)^{4},
$$

which by definition of $a_{n}$ equals $1-\left(1-a_{2 n}\right)^{4}$. This, together with (3.9) completes the proof of the claim.

Lemma 3.5. If $\delta$ is such that the sequence $\left(b_{n}\left(p_{c}, \delta\right)\right)$ is bounded away from 1 , then $\exists \varepsilon>0$ s.t. the family $\left(b_{n}(p, \varepsilon), n=1,2, \cdots, p \in\left[p_{c}, p_{c}+\varepsilon\right]\right)$, is bounded away from 1 .

Proof. Take $\varepsilon$ so small that $\left(p_{c}+\varepsilon\right)+\left(1-p_{c}-\varepsilon\right) \varepsilon<p_{c}+\left(1-p_{c}\right) \delta$, and apply Corollary 2.4.

Lemma 3.6. If $\delta$ is such that the sequence $a_{n}(\delta)$ is bounded away from 1 , then, with $\varepsilon$ as in the previous lemma,

$$
\theta(p, \varepsilon)=0, p \in\left(p_{c}, p_{c}+\varepsilon\right] .
$$

Remark: in fact we prove the following stronger claim: Let $\delta$ be such that the sequence $\left(a_{n}(\delta)\right)$ is bounded away from 1 . Then, with $\varepsilon$ as in Lemma 3.5,

$$
\exists \alpha>0 \text { s.t. } \forall p \in\left(p_{c}, p_{c}+\varepsilon\right] \exists C \forall n \mathcal{P}_{p, \varepsilon}(O \rightarrow S(n)) \leq C n^{-\alpha} .
$$

Proof. Let $\delta$ as in the statement of the claim. Then, by the previous Lemma we can find a $b<1$ such that, for all $p \in\left[p_{c}, p_{c}+\varepsilon\right]$,

$$
b_{n}(p, \varepsilon)<b .
$$

Let $n \geq 1$ and $p \in\left(p_{c}, p_{c}+\varepsilon\right]$. Let $C_{1}$ be a positive constant (at the moment we take $C_{1}$ arbitrary; later we will choose a suitable value, depending on $p$ ). Define the random variables $X_{i}, X_{i}^{*}, Y_{i}$ and $Z_{i}$ as before (so, the $X_{i}$ 's are i.i.d. Bernoulli with parameter $p$, the $Y_{i}$ 's i.i.d. Bernoulli with parameter $\varepsilon$ etc.). In addition, we now take

$$
X_{i}^{(* c)}=I\left(X_{i}=1 \text { but its } X \text {-occupied cluster contains no contour around } B\left(C_{1} \log n\right),\right.
$$

and

$$
Z_{i}^{(c)}=X_{i}^{(* c)} \vee Y_{i}
$$

Note that if there is an infinite $X$-occupied path from $S\left(C_{1} \log n\right)$ then (since this path intersects every contour around $\left.B\left(C_{1} \log n\right)\right)$ we have $X_{i}^{*} \leq X_{i}^{(* c)}$ for all $i \in \mathbb{Z}^{2} \backslash B\left(C_{1} \log n\right)$. So we have

$$
\begin{aligned}
& \mathcal{P}_{p, \varepsilon}(O \rightarrow S(n)) \\
= & \mathcal{P}(\exists Z-\text { occupied path from } O \text { to } S(n)) \\
\leq & \mathcal{P}\left(\exists Z-\text { occupied path from } S\left(C_{1} \log n\right) \text { to } S(n)\right) \\
\leq & \mathcal{P}\left(\exists Z^{(c)}-\text { occupied path from } S\left(C_{1} \log n\right) \text { to } S(n)\right) \\
+ & \mathcal{P}\left(\nexists \text { infinite } X-\text { occupied path from } S\left(C_{1} \log n\right)\right) .
\end{aligned}
$$


From standard percolation theory we know that the last term in the r.h.s. of (3.12) is at most

$$
C_{2}(p) \exp \left(-\beta_{1}(p) C_{1} \log n\right)
$$

where $C_{2}(p)$ and $\beta_{1}(p)$ are positive constants which depend only on $p$.

Now we handle the first term in the r.h.s. of (3.12). Let $K$ denote the set of all positive even integers $k$ with $C_{1} \log n<3^{k}<5 \times 3^{k}<n$, and consider the annuli $A\left(3^{k}, 5 \times 3^{k}\right), k \in K$. These annuli are pairwise disjoint. By this, and the construction, we have that the first term in the r.h.s. of (3.12) is at most

$$
\prod_{k \in K} b_{3^{k}}(p, \varepsilon)<b^{|K|}
$$

where the last inequality comes from (3.11) and where $|K|$ denotes the size of $K$. From the definition of $K$ it is clear that there is a $C_{3}>0$ (which is a function of $C_{1}$ ), and a $\beta_{2}>0$ (which is a constant which does not depend on $p$ ) s.t. (3.14) is at most $C_{3} n^{-\beta_{2}}$.

So for all $C_{1}>0$, we have a $C_{3}>0$ so that for all $p \in\left(p_{c}, p_{c}+\varepsilon\right)$ and for all sufficiently large (depending on $\left.C_{1}\right) n$,

$$
\begin{aligned}
& \mathcal{P}(\exists Z-\text { occupied path from } O \text { to } S(n)) \\
\leq \quad & C_{2}(p) \exp \left(-\beta_{1}(p) C_{1} \log n\right)+C_{3} n^{-\beta_{2}} .
\end{aligned}
$$

The claim now follows by choosing $C_{1}$ so large (depending on $p$ ) that $C_{1} \beta_{1}(p) \geq \beta_{2}$, and then finally choosing the multiplicative constant so large that the inequality holds for all $n$.

The theorem now follows easily by combining this lemma with (3.2).

\section{THE PERMANENT SELF-DESTRUCTIVE PROCESS}

\section{1 background}

Informally, consider the following process in time on the square lattice: at time 0 each site is vacant. All the time, vacant sites become occupied at rate 1. But whenever an infinite occupied cluster occurs, it is immediately destroyed (that is, all its sites become vacant) after which its sites again become occupied at rate 1, as before. An interesting question is whether this description makes sense. Motivation comes partly from forest fire models on a finite box in $\mathbb{Z}^{2}$ : As above, initially everything is vacant and sites become occupied (by a tree) at rate 1 . At each tree, ignition attempts are made at rate $\varepsilon$, independent of the growth process. If, at the time of an ignition attempt, the corresponding site is occupied, the tree is set on fire. Finally, a burning tree burns for an exponentially distributed time with mean $\delta$, and during that time it spreads the fire to adjacent trees at rate $\lambda$. After a tree is burned down the site is vacant and remains vacant for an exponentially distributed (mean 1) time etc. Special attention in the literature is given to the study of what happens (to the equilibrium distribution) when one lets (in some suitable way) the box size and the fire spread rate $\lambda$ go to $\infty$, and $\varepsilon$ and $\delta$ approach 0 . It seems (but most of the results are non-rigorous) that this leads to so-called self-organised critical behaviour (see e.g. Jensen (1998)). From a mathematical point of view it is natural to ask whether one can still define a process when, in some sense, we take all the parameters equal to the above mentioned limit values. Now, one might interpret an ignition rate $\varepsilon=0$ by saying that only infinite clusters are ignited. And a natural interpretation of $\lambda \rightarrow \infty$ and $\delta \rightarrow 0$ could be that the entire cluster is burned instantaneously. These considerations lead to the question above. This question is also closely related to a problem posed by Aldous (2000). An analog of this question for a 1-dimensional system was answered affirmatively in Theorem 1 by Van den Berg and Tòth 
(2001). That paper also gives some further background and relation with other problems. Formally, our question is as follows:

\section{Question}

Do there exist processes $\chi_{i}: \mathbb{R}_{+} \mapsto\{0,1\}, i \in \mathbb{Z}^{2}$, defined jointly on the same probability space, with the following properties:

(a) Almost surely, for all $i \in \mathbb{Z}^{2}, \chi_{i}(0)=0$.

(b) Almost surely, for all $i \in \mathbb{Z}^{2}, t \mapsto \chi_{i}(t)$ is continuous from the right having left limits (c.a.d.l.a.g.).

(c) Let $U_{i}^{(k)}$ denote the length of the $k$ th interval during which $\chi_{i}(\cdot)$ equals 0 . Then the random variables $\left(U_{i}^{(k)}\right)_{i \in \mathbb{Z}^{2}, k \in \mathbb{N}}$ are independent, exponentially distributed with mean 1 .

(d) Almost surely, for all $t \in \mathbb{R}_{+}$and $i \in \mathbb{Z}^{2}$ with $\chi_{i}\left(t^{-}\right)=1$ : if there is an infinite path $\pi$ from $i$ with $\chi_{j}\left(t^{-}\right)=1$ for all $j \in \pi$, then $\chi_{i}(t)=0$, else $\chi_{i}(t)=1$.

Theorem 4.1. If Conjecture 3.2 is true, then there are no processes $\chi_{i}: \mathbb{R}_{+} \mapsto\{0,1\}, i \in \mathbb{Z}^{2}$, with properties (a)-(d) above.

Before we prove this theorem we state the following lemma. First a definition:

Let $k \in \mathbb{N}$ and $\delta>0$. Suppose initially all sites (of the square lattice) are independently open with probability $p_{c}$, after which we make vacant all sites whose occupied cluster contains a contour around the box $B(k)$. Finally (as in the definition of $\mathcal{P}_{p, \delta}$ ) we give each vacant site a $\delta$-enhancement. Let $\mu_{\delta}^{(k)}$ denote the distribution of the final configuration.

Lemma 4.2. If $\delta$ is such that the sequence $\left(a_{n}(\delta)\right)$ is bounded away from 1 , then

$$
\forall k \mu_{\delta}^{(k)}(\exists \text { an infinite occupied cluster })=0 .
$$

Proof. The proof of this lemma is completely similar to(and, in fact, simpler than) that of Theorem 3.3 in the previous section, and therefore we omit it.

\subsection{Proof of theorem 4.1}

Proof. Suppose we have $\delta>0$ such that the sequence $\left(a_{n}(\delta), n \in \mathbb{N}\right)$ is bounded away from 1 . Assume we do have processses $\chi_{i}(\cdot)$ with the properties (a)-(d). We will show that this leads to a contradiction. Let the $U_{i}^{(k)}$ 's be as in property (c).

Now first consider, for each $m$, the following process: initially all sites are vacant. Vacant sites become occupied at rate 1 . At time $t_{c}$ (defined by the relation $1-\exp \left(-t_{c}\right)=p_{c}$ ), all sites which have an occupied path to an occupied contour around $B(m)$, become vacant. After that, vacant sites become occupied again at rate 1 . Let $\eta_{i}^{(m)}(t)$ be the state of site $i$ at time $t$. All these processes with different $m$ can be simply coupled together (and with the $\chi$ processes) by using the $U$ variables mentioned above. In fact, for this coupling we only use the $U^{(1)}$ 's and $U^{(2)}$ 's exactly in the same way as in the Lemma 2.3 (with the $T$ now equal to $t_{c}$, and with an obvious modification of 'catastrophe'): a site $i$ is initially vacant and becomes occupied at time $U_{i}^{(1)}$. If this time is larger than $t_{c}$, it then remains occupied forever. However, if at time $t_{c}$ its occupied cluster contains a contour around $B(m)$, it becomes vacant, and remains vacant until time $t_{c}+U_{i}^{(2)}$, after which it is occupied forever. It is clear that, for $t>t_{c}$, the collection $\left(\eta_{i}^{(m)}(t), i \in \mathbb{Z}^{2}\right.$ ) has distribution $\mu_{\delta}^{(m)}$ (defined above Lemma $4.2)$ with $\delta=1-\exp \left(-\left(t-t_{c}\right)\right)$. Hence, by that lemma (and the assumption in the beginning of this proof), and because each $\eta_{i}^{(m)}(t)$ is increasing in $t>t_{c}$, we have

$$
\begin{aligned}
& \exists \tau>0 \text { such that } \forall m \\
& \mathcal{P}\left(\exists \text { infinite } \eta^{(m)}(t) \text {-occupied cluster for some } t<t_{c}+\tau\right)=0 .
\end{aligned}
$$




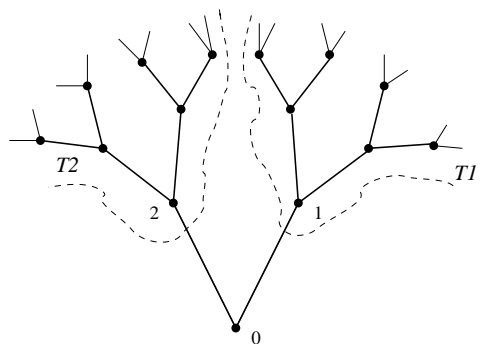

Figure 2: The binary tree

Now back to the $\chi_{i}(t)$ 's. Let $\tau$ be as in (4.1). Clearly (by property (a) and (c)), with probability 1 , there is no site $i$ and $t \leq t_{c}$ with $\chi_{i}\left(t^{-}\right)=1$ and $\chi_{i}(t)=0$. If, in addition, there is no $t \in\left(t_{c}, t_{c}+\tau\right)$ and site $i$ with $\chi_{i}\left(t^{-}\right)=1$ and $\chi_{i}(t)=0$, then $\chi_{j}(t)=I\left(U_{j}^{(1)}<t\right), j \in \mathbb{Z}^{2}, t \in\left(t_{c}, t_{c}+\tau\right)$. However, with probability 1 , for all $t>t_{c}$ there is an infinite cluster of $j \in \mathbb{Z}^{2}$ with $U_{j}^{(1)}<t$. This gives a contradiction with property (d). So, almost surely, there is a $t \in\left(t_{c}, t_{c}+\tau\right)$ and a site $i$ s.t. $\chi_{i}\left(t^{-}\right)=1$ and $\chi_{i}(t)=0$. And hence,

$$
\exists \hat{t} \in\left(t_{c}, t_{c}+\tau\right) \text { s.t. } \mathcal{P}\left(\exists i \in \mathbb{Z}^{2} \text { and } s \in\left(\hat{t}, t_{c}+\tau\right) \text { with } \chi_{i}\left(s^{-}\right)=1, \chi_{i}(s)=0\right)>0 .
$$

Fix such $\hat{t}$. Now, with exactly the same argument we can prove that, almost surely, the following event occurs:

$$
\exists s \in\left(t_{c},\left(t_{c}+\hat{t}\right) / 2\right) \text { and an } i \in \mathbb{Z}^{2} \text { with } \chi_{i}\left(s^{-}\right)=1, \chi_{i}(s)=0 .
$$

But if that event occurs, then, according to the required properties of the $\chi$ process, there is an infinite path on which the $\chi$ value changes from 1 to 0 at that time $s$. But such path intersects, for some $n$, all contours around $B(n)$. By similar arguments as in the proof of Lemma 2.3 and Lemma 3.6 we conclude that,

$$
\text { a.s. } \exists m \text { s.t. } \chi_{j}(t) \leq \eta_{j}^{(m)}(t) \text {, for all } j \in \mathbb{Z}^{2} \text { and } t>\left(t_{c}+\hat{t}\right) / 2 \text {. }
$$

Combining (4.1) and (4.4) contradicts (4.2).

\section{THE BINARY TREE}

5.1

Now we turn our attention to the binary tree $\mathcal{T}$. This is the infinite tree in which one site, which we call the root, has two neighbours, and all other sites have three neighbours (see figure 2). The root will simply be denoted by 0 and its two neighbours by 1 and 2 respectively. The sites 1 and 2 can be considered as roots of the subtrees $\mathcal{T}_{1}$ and $\mathcal{T}_{2}$ respectively.

For ordinary site percolation on $\mathcal{T}$ with parameter $p$ it is well-known that the critical probability equals $p_{c}=1 / 2$ and that for $p \geq 1 / 2$

$$
\theta(p):=\mathcal{P}_{p}(0 \rightarrow \infty)=\frac{2 p-1}{p} .
$$

In particular $\theta\left(p_{c}\right)=0$. 
In fact, using that $\mathcal{T}_{1}$ and $\mathcal{T}_{2}$ are copies of $\mathcal{T}$ one has that $\theta(p)$ satisfies

$$
\theta(p)=p\left(1-(1-\theta(p))^{2}\right) .
$$

Remark: Moreover, if $p>p_{c}, \theta(p)$ is the unique positive solution of the equation $x=p\left(1-(1-x)^{2}\right)$. We will come back to this equation later.

Now we study the probability measures $\mathcal{P}_{p, \delta}$ and the function $\theta(p, \delta)=\mathcal{P}_{p, \delta}(0 \rightarrow \infty)$. We will show that for $\mathcal{T}$, the answer to the Question below Proposition 3.1 is positive, i.e. that for $\mathcal{T}$ (3.3) holds. This in contrast to what we conjecture for the square lattice.

In fact we prove something stronger, namely:

Theorem 5.1. We have

(i) For all $\delta>0$, there exists a $\hat{p}>p_{c}$ and $\alpha>0$ such that for all $p \in\left[p_{c}, \hat{p}\right], \theta(p, \delta)>\alpha$.

(ii) Moreover,

$$
\lim _{p \downarrow p_{c}} \theta(p, \delta)=\theta\left(p_{c}, \delta\right)\left(=\theta\left(p_{c}+\left(1-p_{c}\right) \delta\right)>0\right) .
$$

Remark: From this theorem it is easy to obtain similar results for the Bethe lattice $\mathcal{B}$ (the tree where each site has exactly three neighbours). That graph is in fact nicer because all sites are 'equivalent'. Note that if we delete a site and its three edges from $\mathcal{B}$, three separate copies of $\mathcal{T}$ are left. For ordinary percolation this gives immediately that the probability that a given site is in an infinite occupied cluster equals $p$ (the probability that the site itself is occupied) times the probability that at least one of its three neighbours is in an infinite occupied cluster in its corresponding copy of $\mathcal{T}$. So

$$
\theta^{\mathcal{B}}(p)=p\left(1-\left(1-\theta^{\mathcal{T}}(p)\right)^{3}\right) .
$$

In particular $\mathcal{B}$ has the same critical probability $1 / 2$ as $\mathcal{T}$. For our model 'with destruction' the situation is more complicated, but we can still immediately get an inequality as follows. Using the description with $X$ and $Y$ variables in Section 2, it is clear that if a site $i$ has $X_{i}=0$, then each infinite $X$-occupied cluster belongs to exactly one of the three above mentioned copies of $\mathcal{T}$. A few seconds thought then yields

$$
\theta^{\mathcal{B}}(p, \delta) \geq(1-p) \delta\left(1-\left(1-\theta^{\mathcal{T}}(p, \delta)\right)^{3}\right) .
$$

(The factor $\delta$ comes from the fact that $Y_{i}$ has to be 1 when $X_{i}=0$ and $Z_{i}=1$ ). This, combined with part (i) of Theorem 5.1, immediately gives an analog for $\mathcal{B}$ of that part of the theorem. The analog for $\mathcal{B}$ of the second part of the theorem can then be obtained from the analog for $\mathcal{B}$ of the first part, in exactly the same way in which we will derive the second part for $\mathcal{T}$ from the first part for $\mathcal{T}$.

\subsection{Proof of Theorem 5.1}

Proof. As for ordinary percolation it is natural to use the fact that $\mathcal{T}_{1}$ and $\mathcal{T}_{2}$ are copies of $\mathcal{T}$. The removal of infinite clusters introduces dependencies between these copies, which complicates things. By defining suitable classes of random variables these complications can be handled quite smoothly: Let $X_{i}, i \in V(\mathcal{T})$, and $Y_{i}, i \in V(\mathcal{T})$ be as in the beginning of Section 2. (So the $X_{i}$ 's are i.i.d. Bernoulli with parameter $p$, the $Y_{i}$ 's i.i.d. Bernoulli with parameter $\delta$, and the family of $X_{i}$ 's is independent of the family of $Y_{i}$ 's). We also define the $X_{i}^{*}$ and $Z_{i}, i \in V(\mathcal{T})$ as in Section $2($ with $\Gamma=\infty)$. In addition we define, for our purpose,

$$
X_{i}^{*+}=\left\{\begin{array}{l}
1 \text { if } X_{i}=1 \text { and there is no } X \text { - occupied path from } i \text { to } \infty \text { or to } 0 \\
0 \text { otherwise. }
\end{array}\right.
$$


It is clear from this definition that $X_{0}^{*+}=0$.

We also introduce the 'natural analogs' of these variables for $\mathcal{T}_{1}$ and $\mathcal{T}_{2}$ :

$X_{i}^{*(1)}, i \in V\left(\mathcal{T}_{1}\right)$ is defined by

$$
X_{i}^{*(1)}=\left\{\begin{array}{l}
1 \text { if } X_{i}=1 \text { and there is no } X \text { - occupied path in } \mathcal{T}_{1} \text { from } i \text { to } \infty \\
0 \text { otherwise. }
\end{array}\right.
$$

$X_{i}^{*+(1)}, i \in V\left(\mathcal{T}_{1}\right)$ is defined by

$$
X_{i}^{*+(1)}=\left\{\begin{array}{l}
1 \text { if } X_{i}=1 \text { and there is no } X \text { - occupied path in } \mathcal{T}_{1} \text { from } i \text { to } \infty \text { or } 1 \\
0 \text { otherwise. }
\end{array}\right.
$$

Further,

$$
Z_{i}^{(1)}=X_{i}^{*(1)} \vee Y_{i}, i \in V\left(\mathcal{T}_{1}\right)
$$

and

$$
Z_{i}^{+(1)}=X_{i}^{*+(1)} \vee Y_{i}, i \in V\left(\mathcal{T}_{1}\right) .
$$

Finally, $X_{i}^{*(2)}, X_{i}^{*+(2)}, Z_{i}^{(2)}$ and $Z_{i}^{+(2)}, i \in V\left(\mathcal{T}_{2}\right)$ are defined analogously.

Remark: From the fact that $\mathcal{T}_{1}$ is a copy of $\mathcal{T}$ one gets that the probability that 1 is on an infinite $Z^{(1)}$-occupied path in $\mathcal{T}_{1}$ is equal to the probability that 0 is on an infinite $Z$-occupied path (which by definition is $\theta(p, \delta))$.

To get some feeling for these random variables, note that if $X_{i} \vee Y_{i}=1$ and the $X$-occupied cluster of $O$ is finite, and the event ( 1 is on an infinite $Z^{(1)}$-occupied path in $\mathcal{T}_{1}$ or 2 is on an infinite $Z^{(2)}$ occupied path in $\mathcal{T}_{2}$ ) occurs, then 0 is on an infinite $Z$-occupied path. This (see the remark above) easily gives:

$$
\theta(p, \delta) \geq(p+(1-p) \delta)\left(1-(1-\theta(p, \delta))^{2}\right)-\theta(p)
$$

Even simpler to see is the following upper bound:

$$
\theta(p, \delta) \leq(p+(1-p) \delta)\left(1-(1-\theta(p, \delta))^{2}\right)
$$

When $p$ is larger than but very close to $p_{c}$, then $\theta(p)$ is close to 0 so that both (5.7) and (5.8) are close to the equation (5.2) (with $\theta(p+(1-p) \delta$ ) instead of $\theta(p))$. One would like to conclude from this that if $p \downarrow p_{c}$, then $\theta(p, \delta) \rightarrow \theta\left(p_{c}+\left(1-p_{c}\right) \delta\right.$ ) (which as we observed earlier equals $\theta\left(p_{c}, \delta\right)$ ), so that we get part (ii) of the theorem. The trouble is of course that when we take 0 for $\theta(p, \delta)$ the above inequalities are also satisfied. However, if we can prove that $\liminf _{p \downarrow p_{c}} \theta(p, \delta)>0$, then the above argument works (using (5.2) and the remark following that equation), and we get part (ii) of the theorem. The positivity of this liminf follows immediately from part (i) which we will prove now:

Let, for $n \geq 1, A_{n}$ be the event that there is a $Z$-occupied path of length $n$ from $O$, and $B_{n}$ the event that there is a $Z^{+}$-occupied path of length $n$ from 0 . Let $E$ be the event that the $X$-occupied cluster of $O$ is infinite. Similarly $A_{n}^{(j)}, B_{n}^{(j)}$ and $E^{(j)}$ are defined for $j=1,2$, in the obvious way. For instance, $B_{n}^{(1)}$ is the event that there is a $Z^{+(1)}$-occupied path of length $n$ from 1 in $\mathcal{T}_{1}$. We consider, for each $n$, the probabilities of the combinations of occurrence or non-occurrence of these events: 


$$
\begin{aligned}
& f_{1}(n):=\mathcal{P}\left(\neg E, \neg A_{n}, \neg B_{n}\right) . \\
& f_{2}(n):=\mathcal{P}\left(E, \neg A_{n}, \neg B_{n}\right) . \\
& f_{3}(n):=\mathcal{P}\left(\neg E, A_{n}, B_{n}\right) . \\
& f_{4}(n):=\mathcal{P}\left(\neg E, A_{n}, \neg B_{n}\right) . \\
& f_{5}(n):=\mathcal{P}\left(E, A_{n}, B_{n}\right) .
\end{aligned}
$$

These probabilities depend of course on $p$ and $\delta$ but we have omitted these in our notation. One might expect eight combinations, but some do not occur ( because, for instance, $B_{n}$ implies $A_{n}$ ). One can check that, for $p>p_{c}$ and $\delta>0$, each of $f_{1}(n), \cdots f_{5}(n)$ is positive and that their sum equals 1 .

Also note that, for fixed $n$, the occurrences/non-occurrences of $A_{n+1}, B_{n+1}, E$ are completely determined by those of $\left(A_{n}^{(1)}, B_{n}^{(1)}, E^{(1)}\right),\left(A_{n}^{(2)}, B_{n}^{(2)}, E^{(2)}\right)$ and $X_{0}$ and $Y_{0}$. To illustrate this, suppose the events $\neg E^{(1)}, \neg A_{n}^{(1)}, \neg B_{n}^{(1)}$ occur and also the events $\neg E^{(2)}, A_{n}^{(2)}, \neg B_{n}^{(2)}$, and $X_{0}=1$. This has probability $p f_{1}(n) f_{4}(n)$. It is not hard to check that then $\neg E, A_{n+1}$ and $\neg B_{n+1}$ occur. The same holds when we exchange the roles of $\mathcal{T}_{1}$ and $\mathcal{T}_{2}$. This gives a contribution $2 p f_{1}(n) f_{4}(n)$ to $f_{4}(n+1)$. Considering all contributions we get

$$
\begin{aligned}
f_{4}(n+1)= & p(1-\delta)\left(2 f_{1} f_{3}(n)+2 f_{1}(n) f_{4}(n)+f_{3}(n)^{2}+2 f_{3}(n) f_{4}(n)+f_{4}(n)^{2}\right) \\
& +p \delta\left(2 f_{1}(n) f_{4}(n)+f_{4}(n)^{2}\right) .
\end{aligned}
$$

It is easy to see from the definitions that the functions $f_{1}(n)$ and $f_{2}(n)$ are increasing in $n$ and that $f_{3}(n)$ and $f_{5}(n)$ are decreasing in $n$. By this monotonicity their limits (as $\left.n \rightarrow \infty\right)$, which we denote by $f_{1}, f_{2}, f_{3}, f_{5}$, exist.

Clearly these limits depend on the values of $p$ and $\delta$. Therefore we will sometimes write $f_{i}(p, \delta)$.

Since the $\operatorname{sum} \sum_{i} f_{i}(n)=1$, also the limit $f_{4}$ of the sequence $f_{4}(n)$ exists. Moreover, the limits satisfy the analog of equation (5.10).

We now proceed with the proof of the theorem.

Let $\delta>0$ be given. Suppose that $p$ is such that $\theta(p, \delta)=0$. Hence $f_{3}(n)+f_{4}(n)+f_{5}(n) \rightarrow 0$, $f_{1}(n) \rightarrow 1-\theta(p)$ and $f_{2}(n) \rightarrow \theta(p)$ as $n \rightarrow \infty$. Choose $\varepsilon>0$ such that $(1+\delta(1-\delta))(1-\varepsilon)>0$. The reason for this choice of $\varepsilon$ will become clear later. Because of the convergence of $f_{1}(n)$ to $1-\theta(p)$, we can find an $n_{0}$ such that $\forall n>n_{0}, f_{1}(n)>1-\theta(p)-\varepsilon$. From (5.10) we get for all $n>n_{0}$ :

$$
f_{4}(n+1) \geq 2 p(1-\delta)\left(f_{3}(n)+f_{4}(n)\right)(1-\theta(p)-\varepsilon)+2 p \delta f_{4}(n)(1-\theta(p)-\varepsilon) .
$$

Now we use

$$
f_{3}(n) \geq \delta \frac{1-p}{p} f_{4}(n)
$$

which can be obtained easily from the definitions.

Applying this to (5.11) we obtain for all $n>n_{0}$,

$$
f_{4}(n+1) \geq(2 p+2(1-\delta) \delta(1-p))(1-\theta(p)-\varepsilon) f_{4}(n) .
$$

If now

$$
(2 p+2(1-\delta) \delta(1-p))(1-\theta(p)-\varepsilon)>1,
$$

we obtain $f_{4}(n+1)>f_{4}(n)$ for all $n>n_{0}$, which together with $f_{4}(n)>0$ for all $n$, contradicts the assumption that, for the chosen value of $p, f_{4}(n) \rightarrow 0$.

At $p=p_{c}=1 / 2$ inequality (5.14) is satisfied (we have chosen our $\varepsilon$ accordingly) and hence, by 
continuity, there is a $\hat{p}>p_{c}$ such that for all $p \in\left[p_{c}, \hat{p}\right]$, the inequality is satisfied. By the above mentioned contradiction we conclude that $\theta(p, \delta)>0$ for $p$ in this interval. Finally, by straightforward 'playing' with the values of $\delta$ and $\hat{p}$ and using Corollary 2.4, part (i) of the theorem follows. As we saw earlier, part (ii) can then be obtained quite easily.

\section{Acknowledgments}

Part of this research was done at the Isaac Newton Institute in the fall of 2002.

We thank Antal Jarai, Ronald Meester, Jeff Steif and Balint Tóth for stimulating discussions on these and related problems, and Adri Steenbeek for writing simulation software.

\section{References}

Aldous, D. (2000), The Percolation Process on a Tree where Infinite Clusters are Frozen. Math. Proc. Cambridge Philos. Soc. 128, 465-477.

van den Berg, J. and Tòth, B. (2001), A signal-recovery system: asymptotic properties, and construction of an infinite-volume process. Stoch. Proc. Appl. 96, 177-190.

Grimmett, G.R. (1999), Percolation, 2nd edition, Grundlehren der mathematischen Wissenschaften 321. (Springer, Berlin).

Jensen, H.J. (1998), self-Organized Criticality, Cambridge Lecture Notes in Physics. 